\title{
An update on the management of acne vulgaris
}

This article was published in the following Dove Press journal:

Clinical, Cosmetic and Investigational Dermatology

10 June 2009

Number of times this article has been viewed

\author{
Jonette Keri ${ }^{1,2}$ \\ Michael Shiman' \\ 'Department of Dermatology \\ and Cutaneous Surgery, University \\ of Miami Miller School of Medicine, \\ Miami, FL, USA; ${ }^{2}$ Dermatology Service, \\ Miami VA Hospital, FL, USA
}

\begin{abstract}
Acne vulgaris is a common skin disorder that can affect individuals from childhood to adulthood, most often occurring in the teenage years. Acne can have a significant physical, emotional, and social impact on an individual. Many different treatment options are available for the treatment of acne vulgaris. Commonly used topical treatments include benzoyl peroxide, antibiotics, sulfur and sodium sulfacetamide, azelaic acid, and retinoids. Systemic treatment is frequently used and includes the use of systemic antibiotics, oral contraceptives, antiandrogens, and retinoids. Other treatment modalities exist such as the use of superficial chemical peels as well as using laser and light devices for the treatment of acne. With the multitude of treatment options and the rapidly expanding newer technologies available to clinicians, it is important to review and be aware of the current literature and studies regarding the treatment of acne vulgaris.
\end{abstract}

Keywords: acne vulgaris, treatment, benzoyl peroxide, antibiotics, retinoids, lasers

\section{Introduction}

Acne is a common skin disorder affecting most individuals in the teenage years but also affecting anyone from childhood to middle age. The treatment for acne dates back to Egyptian times when sulfur was used, to recent times with new forms of treatment with lasers and lights. Why treat acne? Many in the lay community feel that acne is a rite of passage into adulthood and needs no treatment. However, dermatologists can vouch for the physical and emotional scars of acne when left untreated. The psychosocial implications can be huge with serious implications regarding the effect on self-image and the quality of life, so that the impact of acne may be equivalent to that of asthma or epilepsy. ${ }^{1}$ This review will concentrate on prescription-based treatments. A good review of over-the-counter acne treatments can be found in a recent review of these products. ${ }^{2}$

\section{Benzoyl peroxide}

Benzoyl peroxide (BP) has long been the mainstay of treatment for acne. It is available in a variety of over-the-counter and prescription formulations. These formulations may include a number of ingredients that differentiate one product from another. For example, BP may be combined with glycolic acid, urea, or placed into microspheres for a more gentle delivery. It may be used as a wash or leave-on product that may be formulated as a gel or cream. BP washes are easy to use and show efficacy in the $30 \%$ to $40 \%$ range. Such washes are good for truncal acne, as the medication can be
Correspondence: Jonette Keri Department of Dermatology and Cutaneous Surgery, University of Miami School of Medicine, I444 NW 9th Ave., Miami, FL 33 I 36, USA

Tel + I (305) 575-3 I67

Fax + I (305) 575-3125

Email jkeri@med.miami.edu 
dispersed by water while having a shower. Its comedolytic and antibacterial properties are quite useful in the treatment of acne. Antibacterial resistance does not appear to occur with BP, and BP combinations are well documented in decreasing the number of resistant organisms to commonly used antiacne antibiotics by effectively destroying microorganisms before resistance can develop. ${ }^{3}$ When giving long-term oral antibiotics, BP is an important adjuvant drug to employ, as it reduces the number of antibiotic resistant organisms. Concerns in the use of BP include irritation and contact sensitivity. It also has a bleaching property, so that when it is not removed completely, it can often bleach clothing and bedding. ${ }^{4}$ In addition, it can bleach the skin of darker individuals and this must be discussed while initiating therapy in the ethnic population. Recently, combination products have been highly successful in the treatment of acne. Patients have enjoyed the ease of applying only one product that can deliver both BP and the topical antibiotics, erythromycin and clindamycin. In the future, BP may be compounded with other agents as well. In many cases, acne treatment requires a combination therapy, as monotherapy is often less successful. ${ }^{5}$

\section{Topical antibiotics}

In addition to the combination products described above, topical antibiotics alone can be useful in the treatment of acne. Clindamycin and erythromycin work by inhibiting Propionibacterium acnes at the 50S ribosomal subunit where they bind irreversibly to inhibit protein synthesis. $P$. acnes can thrive in an environment that combines sebum and desquamated cells, where it converts sebum to free fatty acids, which then stimulate the body's immune response. Topical antibiotics act against this organism, in addition to having anti-inflammatory properties, making these medications useful in the treatment of acne. ${ }^{6}$ Alone, these medications come in the form of solutions, pledgets, lotions, gels, ointments, and foams. They can be used in such combination products but can also be used in conjunction with BP washes, when leave-on BP is too irritating. Choosing the formulation of the antibiotic is dependent on the patients' lifestyle and skin type. Gels are better suited for oily skin, whereas, lotions and ointments work better on drier skin. For truncal use, foams and specific delivery systems using long armed applicators, such as ClindaReach ${ }^{\circledR}$ (DUSA Pharmaceuticals, Wilmington, MA, USA) offer the advantage of application to difficultto-reach areas of the body. Topical antibiotics, when mixed with BP and systemic agents, make a good combination in treating acne.
Understanding the cost and efficacy of different treatment options is important in deciding which one to choose. A randomized controlled trial studying the cost and efficacy of using BP and topical erythromycin in different regimen combinations concluded that BP as an exclusive treatment option was the most cost-effective option, yet caused more local irritation. In combination with erythromycin, there was less irritation. There was little difference whether erythromycin and benzoyl peroxide were administered separately or as a combined formulation, in terms of efficacy and irritation; however, the drugs applied seperately were three times more cost-effective. ${ }^{7}$

\section{Topical sulfur and sodium sulfacetamide}

Sulfur, used for acne since the time of Cleopatra, is useful as a drying and antibacterial agent. It is available in washes, leave-on lotions, creams, foam formulations, and also as prescription and non-prescription masks. Sulfur can be useful when treating patients who may also have rosacea and/or seborrheic dermatitis, as these conditions can also respond to this medication. Sodium sulfacetamide is often combined with sulfur and has anti-inflammatory properties. When used alone, it can also treat acne and is marketed for the sensitive skin acne patient. ${ }^{8}$

\section{Topical azelaic acid}

Azelaic acid is useful in the treatment of acne and the post-inflammatory pigment changes that often accompany it. Its mechanism of action is by inhibition of protein synthesis, although the exact mechanism is unknown. Though patients often report a burning or stinging sensation with topical application, this usually dissipates in a few (1-4) weeks. This medication can be used daily and increased to twicedaily dosing to allow for some tolerance to build. Azelaic acid has shown to be effective in combination with other topical agents such as benzoyl peroxide, clindamycin gel, erythromycin gel, and tretinoin cream. ${ }^{9}$

\section{Topical retinoids}

The topical retinoids used in the treatment of acne include adapalene, tretinoin, and tazarotene. They are comedolytic and anti-inflammatory. Different formulations aid in decreasing irritation, which is the main side-effect that hinders continued use of this class of drugs. Such medications have been formulated into microsponge delivery systems (Retin-A Micro ${ }^{\circledR}$; OrthoNeutrogena, Skillman, NJ, USA), combined with topical antibiotics and formulated to have 
a more extended release, made into a stable formulation of both solubilized and crystalline tretinoin (Ziana ${ }^{\circledR}$; Medicis Pharmaceutical Corporation Scottsdale AZ, USA), and made into cream rather than gel forms (Differin ${ }^{\circledR}$; Galderma Laboratories, Fort Worth, TX, USA) with the goal of causing less irritation on the skin. Of the topical retinoids, adapalene has the least irritant effect among the three listed above, though it still can cause irritation to the user, even when instructions are followed. A recent review of adapalene cited it as a more stable molecule than tretinoin and one that can be used with $\mathrm{BP}$, as well as during the day because of this stability. ${ }^{10}$ Conversely, another review of a large cohort of patients over 52 weeks found that an aqueous gel formulation of clindamycin $(1.2 \%)$ and a stable combination of tretinoin, which were both crystalline and solubilized, was well tolerated and was effective in treating both inflammatory and noninflammatory lesions. ${ }^{11}$ A novel way of getting around the irritant effect with tazarotene is the short contact method, where, as little as 30 seconds of skin contact resulted in a decrease in acne lesions. ${ }^{12}$ This technique can also be used to increase topical contact time in a gradual fashion. In general, retinoids are considered to be very important in long-term maintenance therapy of acne. Drug companies are looking for ways to improve compliance by decreasing the irritation factor of topical retinoids. The important feature of the retinoids, treating not only the visible but also the invisible lesions must be stressed to the patient. Patients should be given detailed instructions for use, which should include, the amount to be used, the duration that the medication would take to produce visible changes, as well as potential side-effects.

Finally, regarding retinoids, there is one recent reference question on whether the topical form of these medications can lead to systemic side-effects. Though this issue comes up repeatedly, there is very little evidence. Recently, there was a single case report of a 23 -year-old woman using adapalene $0.1 \%$ gel, who developed severe headaches and neck stiffness. ${ }^{13}$ The patient was on no other medications, but was found to have swollen optic discs, although she also had a normal cerebrospinal fluid pressure on lumbar puncture. Six weeks after discontinuation of the adapalene, the discs were back to normal and the headaches were gone. The authors note that the patient had no other risk factors except for being mildly overweight at $70.3 \mathrm{~kg}$ at a height of $164.5 \mathrm{~cm}$.

\section{Systemic antibiotics}

Oral antibiotics are an important treatment option for moderate to severe acne. It was in the 1950 s that oral tetracycline and erythromycin were used to treat acne.
Since then, antibiotics used to treat acne have come to include doxycycline, minocycline, trimethoprim/sulfamethoxazole, amoxicillin, azithromycin, and cephalexin. As with the development of novel formulations for topical acne medications, oral antibiotics have seen some modifications in their formulations as well. Antibiotic dosage for acne has generally followed treatment regimens as for other conditions treated with the same antibiotics. Such doses include, doxycycline $100 \mathrm{mg}$ twice daily or erythromycin $500 \mathrm{mg}$ twice daily. Recently, two novel uses of antibiotics have come to the forefront. The first is by prescribing a sub-MIC (minimal inhibitory concentration) dose, where the antibiotic acts as an anti-inflammatory medication rather than an antibiotic. Doxycycline at $20 \mathrm{mg}$ twice daily is considered to be a subMIC dose. ${ }^{14}$ Another novel dosage/formulation regimen involves using a sustained release form of minocycline which is prescribed in a weight based manner ( $1 \mathrm{mg} / \mathrm{kg} / \mathrm{day})$ with the idea that preventing high levels of the antibiotic in the blood, rapidly after ingestion will lead to less side-effects. This preparation of sustained release minocycline (Solodyn ${ }^{\circledR}$; Medicis Pharmaceutical Corporation, Scottsdale, AZ, USA) has been suggested to have less vestibular side-effects, but has yet to show a decrease in other minocycline induced side effects. Although most dermatologists prefer the tetracycline class of antibiotics when dealing with acne, there is evidence that other antibiotics can be helpful. An alternative dosage plan with azithromycin $500 \mathrm{mg}$ daily, for four days of the month had similar efficacy as doxycycline $100 \mathrm{mg}$ daily. ${ }^{15}$ Multiple other studies using azithromycin as an alternate drug have also shown some positive results. Moreover, a recent study using cephalexin has reported positive results, (49\% of patients were clear of acne or much improved). Although the study design was a retrospective chart review, cephalexin may be an inexpensive alternative when other medications are not available. ${ }^{16}$

There are multiple controversies when it comes to treating acne patients with long-term antibiotics. These include risk of antibacterial resistance and other conditions such as breast cancer, as well as serious autoimmune reactions. Regarding antibacterial resistance, it is important to explain this concept to the patients/caregivers and at the same time employ methods to decrease such resistance. This is as simple as having patients use a combination product of BP and a topical antibiotic, while they are on long-term oral antibiotics. It is important to note that there are some risks to systemic antibiotics with respect to bacterial resistance. In addition to the obvious dermatologic complication of gram-negative acne or folliculitis after long-term oral antibiotics, there is also the risk 
of increased upper respiratory tract infections. Acne patients on oral antibiotics are about two times more likely to develop an upper respiratory tract infection, though their household contacts do not appear to be at an increased risk. ${ }^{17}$

After a 9-year follow-up of 2.1 million women, the observed associations of tetracyclines and macrolides with breast cancer were weak and could be explained by other diseases or factors. ${ }^{18}$

Autoimmune reactions to antibiotics used to treat acne have been associated most often with minocycline. A review of serious adverse reactions to minocycline included autoimmune hepatitis, serum sickness and vasculitis, and a lupus-like syndrome where the complement remains normal, and these serious events appear to be genetically determined by HLAtype, DR4+, DR2+. ${ }^{19}$ A more recent review of patients with minocycline induced autoimmunity (MIA) looked at a 10-year period of patients referred to a tertiary hospital rheumatology service for the treatment of MIA. ${ }^{20}$ This was found to be a very rare entity, accounting for $0.05 \%$ of all children referred to the rheumatology clinic at the tertiary children's hospital. Most autoimmune reactions to minocycline are probably mild and go away undiagnosed. However, of the 27 patients in this study, 7 had persistent chronic autoimmune disease 48 months later, requiring such medications as methotrexate, etanercept and mycophenolate mofetil. There may be a genetic predisposition as it is more common in women, and dermatologists should be aware of this. Doxycyline is also known to trigger severe autoimmune reactions such as reported in a 20-year-old woman on doxycycline for facial acne who presented with fever, lymphadenopathy, hepatitis, nephritis, and severe pneumonitis with respiratory failure. ${ }^{21}$

\section{Oral contraceptives and antiandrogens}

Hormonal manipulation in the treatment of acne came to the forefront in the early 1990s. Currently in the United States there are three oral contraceptive pills approved by the Federal Drug Agency (FDA) for the treatment of acne in women. These include ethinyl estradiol and norgestimate (Ortho Tri-Cyclen ${ }^{\circledR}$; Ortho-McNeil-Janssen Pharmaceuticals, Titusville, NJ, USA), norethindrone acetate and ethinyl estradiol (Estrostep ${ }^{\circledR}$; Warner-Lambert, Morris Plains, NJ, USA) and ethinyl estradiol/drosperinone (YAZ ${ }^{\circledR}$; Bayer, Wayne, NJ, USA). In other countries around the world, cyproterone acetate (Diane-35 ${ }^{\circledR}$; Bayer Pty Ltd, Singapore) is also used for the treatment of acne. The progestin drosperinone, found in products of brands such as $\mathrm{YAZ}^{\circledR}$ and Yasmin $^{\circledR}$ (Bayer, Wayne, NJ, USA), have about the same antiandrogen activity of $25 \mathrm{mg}$ of spironolactone. Although hormonal manipulation will help many patients with acne, it is imperative that dermatologists also be on the lookout for endocrinopathies such as polycystic ovarian syndrome (PCOS). PCOS patients often have irregular menses, hirsutism, acne, infertility, and obesity. However, with this syndrome the patient may not fit the textbook description. A recent review of the evaluation of a patient with suspected hormonal imbalance and acne provides great decision-making algorithms for those treating such patients. ${ }^{22}$ Early identification of such patients can have long-term health benefits, as these patients are more likely to develop insulin resistance and cardiovascular disease than women without PCOS. For patients with PCOS and acne, the ethinylestradiol/drosperinone combination pill can help with both conditions. ${ }^{23}$ In addition, this combination pill $\left(\mathrm{YAZ}^{\circledR}\right.$ ) has been approved for premenstrual dysmorphic disorder (PMDD), a psychiatric diagnosis, associated with severe symptoms prior to menstruation.

Spironolactone is another option for patients with acne. As an antiandrogen, spironolactone has produced good results in the PCOS patient, as well as in the patient without PCOS, but who has menstrual acne flares and deep cystic acne. Spironolactone should not be given to those who have renal impairment. Since it can retain potassium, a good review of other medications the patient is on, is imperative, to exclude potassium retaining medications. Chronic nonsteroidal antiinflammatory medications can also increase potassium and this should be considered when prescribing spironolactone. In addition, this is a category $\mathrm{X}$ drug in pregnancy and thus, the patient should be counseled about this and if there is any risk of pregnancy, it should be evaluated. Dosage ranges for spironolactone are 50-200 mg daily with most prescribers starting at 50-100 $\mathrm{mg}$ a day.

\section{Systemic retinoids}

Isotretinoin is the main systemic retinoid used in the treatment of acne, specifically severe nodulocystic acne. The mechanisms of action of this drug include inhibition of sebaceous gland differentiation with a reduction in sebaceous gland size and sebum production, normalization of follicular epithelial desquamation, and inhibition of neutrophil chemotaxis. Recommended cumulative doses for the treatment of acne are $120-150 \mathrm{mg} / \mathrm{kg}$ with many practitioners using the higher end of this range. Isotretinoin has a variety of adverse effects associated with it. Almost every patient will have xerosis of the skin and chelitis. However, the side-effect of concern with this medication is teratogenicity, which is quite major. 
Up to $50 \%$ of fetuses exposed to this drug during the first trimester will have a major birth defect which includes major cardiovascular, craniofacial, ocular, auditory, central nervous system, and skeletal abnormalities. ${ }^{24}$ In the United States, a federally mandated program, iPledge has been instituted in an attempt to decrease the number of pregnancies while patients are on this medication. This program requires all patients to be registered with an iPledge number and also requires physicians, pharmacists and pharmacy distributors to also be registered if they are involved with the distribution of this drug. Long-term analysis has yet to show if this program decreases the number of pregnancies while on the drug.

Another concern about isotretinoin is whether this medication increases the risk of suicide and depression. A recent review of nine studies evaluating this concern was published. ${ }^{25}$ This systemic review found the rates of depression to be similar among users of isotretinoin and nonusers. The studies examined did not show a statistically significant increase in depression in isotretinoin users before or after treatment, and some showed that the patients had less severe depressive symptoms after treatment. The authors of this review note that all these studies have limitations, but the data do not support a meaningful causative association. However, this association must be discussed with patients upon initiation of this drug and at each follow-up visit.

\section{Superficial chemical peels}

Chemical peeling of the skin has long been used in the treatment of acne. Most peeling for the treatment of acne is superficial. This discussion is focused on the treatment of acne rather than that of acne scarring. In general, most practitioners use salicylic acid, glycolic acid, or trichloroacetic acid for the treatment of acne. Superficial chemical peeling can be done monthly, although some do the peels as often as every 2 weeks and some wait until every 6 weeks. Good patient education is necessary as patients should know what to expect with the peel. A good review of regimens used for patients in office settings is noted in a recent review. ${ }^{26}$ In patients with ethnic skin, salicylic acid remains the peeling agent of choice as the others have been associated with post-inflammatory pigment alteration. A recent comparison of alpha-hydroxy (30\% glycolic acid) versus beta-hydroxy (30\% salicylic acid) peels in a double-blind, randomized study found that both peels were equally effective in the treatment of acne. ${ }^{27}$ However, there were more reported adverse events after the initial treatment with the glycolic acid peel.

\section{Laser and light devices}

With technology progressing at a fast pace, it is not surprising that laser and light therapies would be of interest to those treating acne. Recently, the range of treatments for acne has included photodynamic therapy (PDT), light-emitting diode (LED) therapy and a combination of pneumatic energy and light for the treatment of acne. These therapies work best as adjuvant therapies when combined with traditional therapies.

PDT, which is the treatment of skin with aminolevulinic acid followed by photo activation of the compound, has been shown to help treat acne. ${ }^{28}$ Most patients are able to tolerate the procedure well, but in rare cases PDT has been associated with a painful pustular reaction while being used for the treatment of acne.

Red and blue light therapy is also used to treat acne, with the most recent advances being home-use products that allow the patient to deliver the light therapy at home by a handheld device. Recently, a report of a study using a handheld LED red and blue light device found that the lesion counts decreased throughout the 4-week treatment period and continued for up to 8 weeks post-therapy with the average reduction of $69 \%(\mathrm{p}<0.001) .{ }^{29}$ Similarly, another study looking at acne in patients with type IV skin showed that the red and blue light combination treatment twice weekly for 4 weeks resulted in improvements in noninflammatory and inflammatory lesions. ${ }^{30}$ As inflammatory lesions improved more, the authors felt this was a good treatment particularly for papulopustular acne.

A novel combination product called Isolaz ${ }^{\mathrm{TM}}$ (Aesthera, Pleasanton, CA, USA) which uses a vacuum along with a broad band light source, has been shown to be effective in the treatment of acne. ${ }^{31}$ This small study (11 patients) treated patients with the device at 3-week intervals and had significant decreases in both inflammatory and noninflammatory lesions. Most patients expressed satisfaction with this treatment.

\section{Future treatment options}

Probably the most interesting treatment on the horizon is vaccine therapy for acne. The vaccines are targeted against the $P$. acnes bacteria. As mentioned at the beginning of this article, acne is a multifactorial disease, and thereby targeting one area may not result in eradication of the condition. However, $P$. acnes is involved in diseases other than acne including infectious conditions such as endocarditis, endophthalmitis, osteomyelitis and post-surgery infections, and this has lead researchers to develop vaccines. A recent 
review highlights the latest work on the $P$. acnes vaccines and how they have shown good results and immunity, when used in mice challenged by $P$. acnes. ${ }^{32}$

In conclusion, practitioners should be aware of the traditional therapeutic choices for the treatment of acne, but should work to refine their knowledge about the different formulations of products available to them to prescribe. As to newer technologies, it is imperative that physicians are aware of these modalities and even if they are not actively using them, they should have a working knowledge of them to answer patient questions.

\section{Disclosures}

Dr. Keri has served on an advisory panel and been a speaker for Medicis.

\section{References}

1. Thomas DR. Psychosocial effects of acne. J Cutan Med Surg. 2004; $8: 3-5$.

2. Bowe WP, Shalita AR. Effective over-the-counter acne treatments. Semin Cutan Med Surg. 2008;27:170-176.

3. Eady EA, Farmery MR, Ross JI, et al. Effects of benzoyl peroxide and erythromycin alone and in combination against antibiotic-sensitive and -resistant skin bacteria from acne patients. $\mathrm{Br} J$ Dermatol. 1994;131:331-336.

4. Worret WI, Fluhr JW. Acne therapy with topical benzoyl peroxide, antibiotics and azelaic acid. J Dtsch Dermatol Ges. 2006;4:293-300.

5. Leyden JJ. A review of the use of combination therapies for the treatment of acne vulgaris. J Am Acad Dermatol. 2003;49:200-210.

6. Russell JJ. Topical therapy for acne. Am Fam Physician. 2000; 61:357-366.

7. Ozolins M, Eady EA, Avery A, et al. Randomised controlled multiple treatment comparison to provide a cost-effectiveness rationale for the selection of antimicrobial therapy in acne. Health Technology Assessment. 2005;9:212.

8. Gupta AK, Nicol K. The use of sulfur in dermatology. J Drugs Dermatol. 2004;3:427-431.

9. Webster G. Combination azelaic acid therapy for acne vulgaris. J Am Acad Dermatol. 2000;43:47-50.

10. Irby CE, Yentzer BA, Feldman SR. A review of adapalene in the treatment of acne vulgaris. $J$ Adolesc Health. 2008;43:421-424.

11. Del Rosso JQ, Jitpraphai W, Bhambri S, et al. Clindamycin phosphate $1.2 \%$ - tretinoin $0.025 \%$ gel: vehicle characteristics, stability, and tolerability. Cutis. 2008;81:405-408.

12. Bershad S, Kranjac Singer G, Parente JE, et al. Successful treatment of acne vulgaris using a new method: results of a randomized vehiclecontrolled trial of short-contact therapy with $0.1 \%$ tazarotene gel. Arch Dermatol. 2002;138:481-489.
13. Givre SJ, Fleischman D. Intracranial hypertension in a patient using topical adapalene. J Neuroophthalmol. 2008;28:156-158.

14. Skidmore R, Kovach R, Walker C, et al. Effects of subantimicrobialdose doxycycline in the treatment of moderate acne. Arch Dermatol. 2003;139:459-464.

15. Parsad D, Pandhi R, Nagpal R, et al. Azithromycin monthly pulse vs daily doxycycline in the treatment of acne vulgaris. $J$ Dermatol. 2001;28:1-4.

16. Fenner JA, Wiss K, Levin NA. Oral cephalexin for acne vulgaris: clinical experience with 93 patients. Pediatr Dermatol. 2008;25: 179-183.

17. Bowe WP, Hoffstad O, Margolis DJ. Upper respiratory tract infection in household contacts of acne patients. Dermatology. 2007;215: 213-218.

18. Friedman GD, Oestreicher N, Chan J, et al. Antibiotics and risk of breast cancer: up to 9 years of follow-up of 2.1 million women. Cancer Epidemiol Biomarkers Prev. 2006;15:2102-2106.

19. Dunphy J, Oliver M, Rands AL, et al. Antineutrophil cytoplasmic antibodies and HLA class II alleles in minocycline-induced lupus-like syndrome. Br J Dermatol. 2000;142:461-467.

20. El-Hallak M, Giani T, Yeniay BS, et al. Chronic minocycline-induced autoimmunity in children. J Pediatr. 2008;153:314-319.

21. Robles DT, Leonard JL, Compton N, et al. Severe drug hypersensitivity reaction in a young woman treated with doxycycline. Dermatology. 2008;217:23-26.

22. George R, Clarke S, Thiboutot D. Hormonal Therapy for Acne. $M D$ Semin Cutan Med Surg. 2008;27:188-196.

23. Mathur R, Levin O, Azziz R. Use of ethinylestradiol/drospirenone combination in patients with the polycystic ovary syndrome. Ther Clin Risk Manag. 2008;4:487-492.

24. Lammer EJ, Chen DT, Hoar RM, et al. Retinoic Acid Embryopathy. N Eng J Med. 1985;313:837-841.

25. Marqueling AL, Zane LT. Depression and suicidal behavior in acne patients treated with isotretinoin: a systematic review. Semin Cutan Med Surg. 2007;26:210-220.

26. Kempiak SJ, Uebelhoer N. Superficial chemical peels and microdermabrasion for acne vulgaris. Semin Cutan Med Surg. 2008; 27:212-220

27. Kessler E, Flanagan K, Chia C, et al. Comparison of alpha- and betahydroxy acid chemical peels in the treatment of mild to moderately severe facial acne vulgaris. Dermatol Surg. 2008;34:45-50.

28. Alexiades-Armenakas M. Aminolevulinic acid photodynamic therapy for actinic keratoses/actinic cheilitis/acne: vascular lasers. Dermatol Clin. 2007;25:25-33.

29. Sadick NS. Handheld LED array device in the treatment of acne vulgaris. J Drugs Dermatol. 2008;7:347-350.

30. Lee SY, You CE, Park MY. Blue and red light combination LED phototherapy for acne vulgaris in patients with skin phototype IV. Lasers Surg Med. 2007;39:180-188.

31. Gold MH, Biron J. Efficacy of a novel combination of pneumatic energy and broadband light for the treatment of acne. J Drugs Dermatol. 2008;7:639-642.

32. Nakatsuji T, Rasochova L, Huang CM. Vaccine therapy for P. acnesassociated diseases. Infect Disord Drug Targets. 2008;8:160-165.
Clinical, Cosmetic and Investigational Dermatology

\section{Publish your work in this journal}

Clinical, Cosmetic and Investigational Dermatology is an international, peer-reviewed, open access, online journal that focuses on the latest clinical and experimental research in all aspects of skin disease and cosmetic interventions. All areas of dermatology will be covered; contributions will be welcomed from all clinicians and

\section{Dovepress}

basic science researchers globally. This journal is indexed on CAS The manuscript management system is completely online and includes a very quick and fair peer-review system, which is all easy to use. Visit http://www.dovepress.com/testimonials.php to read real quotes from published authors. 